\title{
Rectifying an IRKsome finding
}

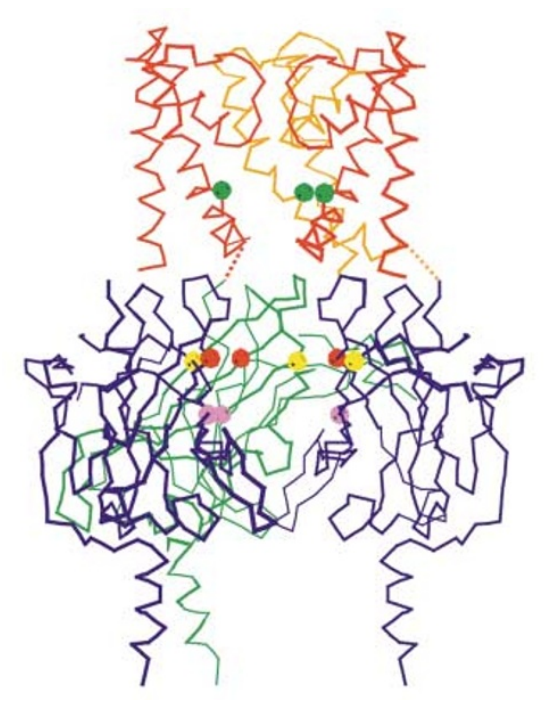

Stereo view of a channel consisting of the transmembrane pore of th MthK channel docked with the cytoplasmic pore of mGIRK1. The front subunit of the tetramer has been removed to provide a clearer view of the pore structure. The coloured spheres indicate positions where mutations affect pon conduction or blocking by pon conduction or blocking by impermeant cations. Reproduced with permission, from Nishida 2
MacKinnon () 2003 Elsevier Science.

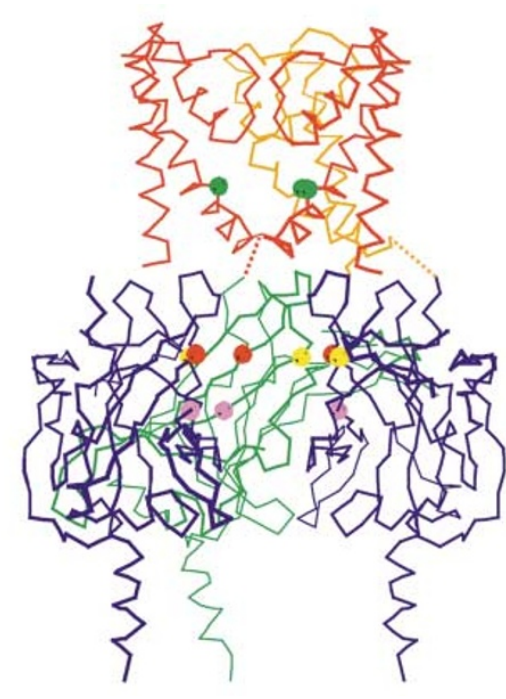

It is easy to envisage ion channels simply as holes in the cell membrane through which ions can pass freely in both directions, and this is indeed the case for many channels. However, there is a subset of $\mathrm{K}^{+}$channels that conduct ions more efficiently into than out of the cell. These are known as inward rectifier $\mathrm{K}^{+}$(IRK) channels. A new study published in
Cell identifies the unique structural features of these channels that account for this property.

IRK channels are important for restoring the resting potential of neurons after electrical activity. During an action potential, $\mathrm{Na}^{+}$ions flow into the neuron, and if all the $\mathrm{K}^{+}$ channels were open, $\mathrm{K}^{+}$ions would be expelled, thereby dissipating the positive charge that is generated inside the neuron. To stop this from happening, the IRK channels become blocked by intracellular cations, such as polyamines, which can bind inside the channel but cannot pass through. As the action potential subsides, the neuron transiently becomes hyperpolarized, and the IRK channels re-open to allow $\mathrm{K}^{+}$ions to enter and restore the cell to its resting potential.

What are the structural properties of the IRK channels that cause them to function as rectifiers? It was suspected that part of the answer lay in the carboxyl terminus, because mutations in this region affected the binding affinity of blockers. Nishida and MacKinnon examined the crystal structure of the amino and carboxyl termini of mGIRK1, a G-proteingated IRK channel from the mouse. Both termini lie inside the cell, and the authors found that they assemble into a tetramer to form a pore-like structure that extends the total length of the channel to nearly $60 \AA$. The pore is lined with negatively charged amino acid side chains, which provide an excellent binding substrate for polyamines.

The efficacy of blocking by polyamines is higher at positive membrane

\section{STEM CELLS}

\section{Cells under surveillance}

Embryonic stem (ES) cells are a promising source of new neurons to replace those that are lost through disease and injury. Functional recovery is the ultimate goal, but the cells must first be targeted to the right place, differentiate appropriately, and become integrated into functional circuits. Previously, the fate of ES cells that were transplanted into the brain could only be assessed retrospectively, through the examination of post-mortem tissue. However, Hoehn et al. have now developed a magnetic resonance imaging (MRI) technique that allows them to follow the movements of grafted cells in the living brain.

In a study reported in the Proceedings of the National Academy of Sciences, the authors induced ischaemia in the right cerebral hemisphere of the rat brain by transiently blocking the middle cerebral artery. They labelled an ES cell line with an MRI contrast agent, grafted these cells into the intact left hemisphere, and tracked their migratory behaviour using MRI. They found that they could detect clusters containing as few as 40 labelled cells - a higher resolution than has previously been achieved with this type of approach.

It has long been suspected that stem cells are preferentially targeted to damaged tissue, and the experiments of Hoehn et al. provide a striking confirmation of this phenomenon. In spite of the relatively large distance between the graft site and the site of brain injury, a large proportion of the grafted ES cells migrated directly across the corpus callosum, which bridges the two hemispheres, and headed straight for the lesioned area. This raises the possibility that ischaemic tissue might release a long-range chemoattractant that directs ES cell migration.

So, Hoehn et al. have developed a highresolution imaging method for tracking the movements of grafted ES cells in the brain. This should prove to be extremely valuable for gauging the success of stem cell transplantation in experimental studies, and perhaps even in the clinic. Also, studying the migratory behaviour of grafted cells might provide some important clues to the nature of the endogenous signals that influence their migration, making it possible to develop more effective transplantation protocols.

Heather Wood

(D) References and links ORIGINAL RESEARCH PAPER Hoehn, M. et al. Monitoring of stem cell migration in vivo: a highly resolved in vivo magnetic resonance imaging investigation of experimental stroke in rat. Proc. Natt Acad. Sci. USA 99, 16267-16272 (2002) FURTHER READING Rossi, F. \& Cattaneo, E. Neural stem FURTHER READING Rossi, F. \& Cattaneo, E. Neural sten
cell therapy for neurological diseases: dreams and reality. Nature Rev. Neurosci. 3, 401-409 (2002) 
potentials. So, blocking is more efficient when the membrane is depolarized. Early measurements implied that the voltage dependence of the blocking reaction is greater than would be predicted from the charge of the blocker alone, and the elongated shape of the mGIRK1 channel provides an explanation for this observation. The length of the channel allows it to accommodate extra $\mathrm{K}^{+}$ions that are expelled outwards when the blockers bind, and this additional charge displacement accounts for the increased voltage dependence of channel block.

Nishida and MacKinnon have shown that structural analysis can provide fascinating insights into the phenomenon of rectification, and it has also enabled them to identify new targets for mutational studies of the IRK channels.

Heather Wood

(2) References and links ORIGINAL RESEARCH PAPER Nishida, M. \& MacKinnon, R. Structural basis of inward rectification: cytoplasmic pore of the G proteingated inward rectifier GIRK1 at $1.8 \AA$ resolution. Cell 111, 957-965 (2003)

FURTHER READING Nichols, C. G. \& Lopatin, A. N. Inward rectifier potassium channels. Annu. Rev. Physiol. 59, 171-191 (1997) WEB SITE

Encyclopedia of Life Sciences: http://www.els.net/

sodium, calcium and potassium channels

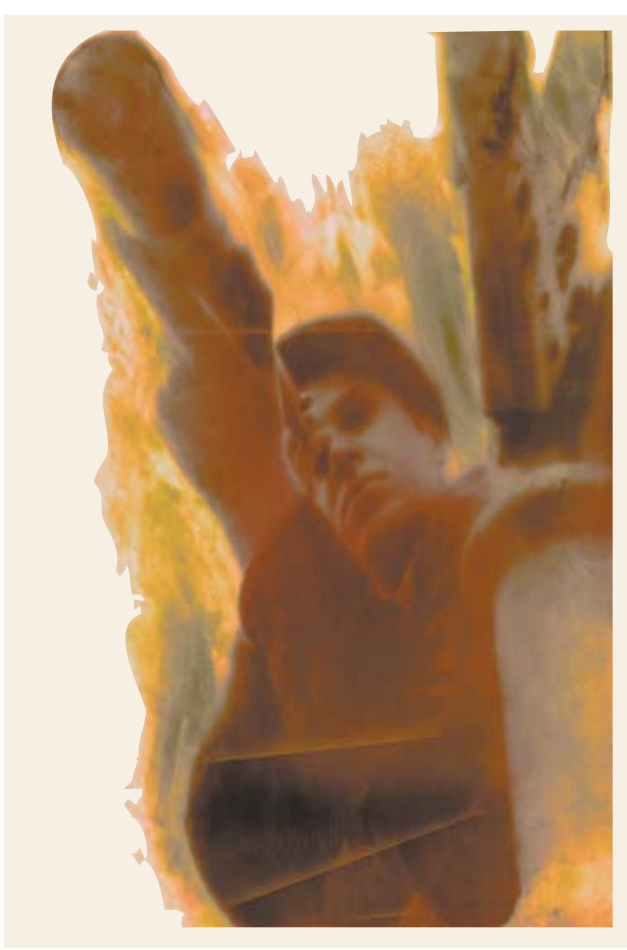

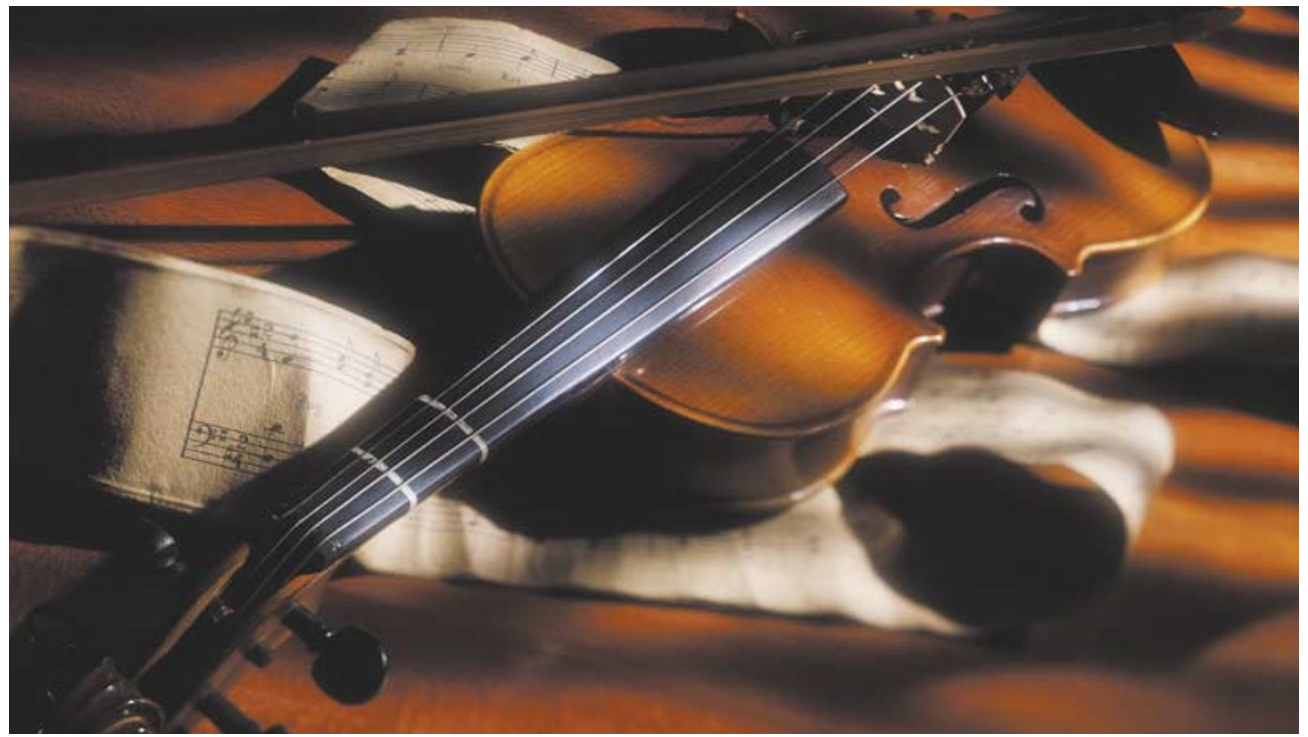

NEUROPHYSIOLOGY

\section{Orchestrating arousal}

How does the brain coordinate the activity of neuronal systems involved in sleep-wake regulation to set our level of arousal? A possible answer to this question was unearthed in the late 1990s, when researchers discovered a neuropeptide system in the lateral hypothalamus that is central to the regulation of arousal states and energy metabolism. Since then, much has been learned about the functions of the hypocretins (also known as orexins), but the cellular mechanisms that govern the activity of hypocretin neurons have received little attention. A recent study by Li et al. shows how the output of this system might be orchestrated at the cellular level.

The hypocretin system makes reciprocal connections with other homeostatic centres within the hypothalamus. Its densest projections outside the hypothalamus are to structures that regulate wakefulness, including the dorsal raphe nucleus (DRN) and the locus coeruleus (LC); hypocretins increase wakefulness and suppress rapid eye movement (REM) sleep. So, the hypocretin system seems to provide a link between our homeostatic needs and our level of arousal.

$\mathrm{Li}$ and colleagues reasoned that there must be some orchestration of output from cells that regulate arousal; this would explain why neurons in other regions of the brain, including the DRN and the LC, show changes in group activity across the sleep-wake cycle. They made

electrophysiological recordings from hypocretin neurons of the lateral hypothalamus, identified in transgenic mouse brain slices by their expression of green fluorescent protein. Hypocretin neurons responded to hypocretins 1 and 2 with a depolarization and excitation that depended on an increase in the synaptic release of glutamate; no direct effects of hypocretin on the membrane properties of these cells were detected. So, the hypocretin system seems to employ a positive feedback loop that involves the activation of a glutamatergic interneuron.
Li et al. then looked for a factor that might reduce the activity of hypocretin cells. In view of the fact that hypocretins have excitatory effects on serotonergic neurons of the DRN and on noradrenergic neurons of the LC, the authors examined the effects of serotonin and noradrenaline on hypocretin neurons. These transmitters directly hyperpolarized and inhibited hypocretin cells. So, negative feedback from REM-off cells of other arousal systems might suppress the output of hypocretin neurons.

How, then, do the hypocretins exert their effects on other neuronal systems? In a recent experiment, Follwell and Ferguson examined the actions of hypocretin on the membrane properties of neurons of the rat paraventricular nucleus of the hypothalamus (PVN). The PVN receives a dense projection from the hypocretin system, and it is well positioned to mediate the effects of hypocretins on autonomic and endocrine functions. The authors found that most magnocellular neurons were depolarized by hypocretin 1, apparently through the activation of a glutamatergic interneuron. This peptide depolarized parvocellular neurons by the enhancement of a non-selective cation conductance.

These studies remind us that, although considerable advances have been made in our understanding of the hypocretin system, further studies are needed to describe the cellular events that underpin its interactions with other systems, its role in regulating arousal states, and its involvement in sleep disorders such as narcolepsy. Rebecca Craven, Senior Subeditor, Nature

\section{(1) References and links}

ORIGINAL RESEARCH PAPERS $L i$, Y. et al. Hypocretin/orexin excites hypocretin neurons via a local glutamate neuron - a potential mechanism for orchestrating the hypothalamic arousal system. Neuron 36, 1169-1181 (2002) | Follwell, M. J. \& Ferguson, A. V. Cellular mechanisms of orexin actions on paraventricular nucleus neurones in rat hypothalamus. J. Physiol. (Lond.) 545, 855-867 (2002) FURTHER READING Sutcliffe, J. G. \& de Lecea, L. The hypocretins: setting the arousal threshold. Nature Rev. Neurosci. 3, 339-349 (2002) 